\title{
An Application of Active Frequency Selective Surface to Reconfigurable Antenna Technology
}

\author{
B. Sanz-Izquierdo, B. Liang, E. A. Parker and J. C. Batchelor \\ School of Engineering and Digital Arts, The University of Kent, Canterbury, United Kingdom, \\ b.sanz@kent.ac.uk
}

\begin{abstract}
This paper illustrates the use of active frequency selective surfaces (FSS) in reconfigurable antennas. A patch-form dual-polarized tunable FSS is adapted for use as an active artificial magnetic conductor (AMC). As with the tunable FSS, the active AMC offers independent tuning capability of the reflection phases for two polarisations of incident EM waves. By combining the AMC with a wideband coplanar waveguide fed monopole antenna, circularly polarisation $(\mathrm{CP})$ capability is realized at any frequency over the 1.15-1.60GHz band. Furthermore, the CP state can be switched between left hand $\mathrm{CP}$ and right hand $\mathrm{CP}$, by simply interchanging the capacitances of varactors controlling the reflection phases of the two orthogonal polarised waves. The antenna covers the frequency bands of all operational and in-preparation satellite navigation systems, including GPS, GLONASS, Beidou and Galileo. polarization reconfigurable circular polarized (CP) antenna using active artificial ground (AG) structure
\end{abstract}

Index Terms- $\mathrm{CP}$ antenna, frequency tunable, polarization switchable, active aritificial ground, satellite navigation.

\section{INTRODUCTION}

Satellite navigation systems (SNS) are regarded as one of the most successful products for wireless communications. They have been widely used in military, commercial and civil applications. Based on the capability of identifying user locations, SNS provide position-related services and related technologies. Currently, the most well-known satellite navigation system is Global Positioning System (GPS). It is capable of providing real-time and all-weather global navigation services for the areas of land, sea and air. The main drawback is that it is maintained by a single country, which raises concerns from other countries, In order to increase national security, some other navigation systems are also in use or under preparation, such as GLONASS of Russia, Galileo of European Union, and Beidou of China.

Circular polarized (CP) antennas are employed by SNS to transmit signals. These types of antennas have advantages such as the alleviation of multi-path interferences or fading, insensitivity to Faraday rotation effect, and no strict requirement of antenna orientations [1]. Wideband or multiband CP antennas for SNS have drawn increasing interest [2-4]. However, if one or more SNS are not required and need to be suspended, passive antennas are not suitable due to their fixed characteristics and absence of frequency selection.

Artificial magnetic conductors (AMC) structures are a promising candidate to realize $\mathrm{CP}$ antennas. They are low profile and avoid the use of impedance matching networks $[5,6]$. AMC structures are electromagnetic band gap (EBG) structures that can be fabricated without vias, hereby losing the ability of surface wave suppression but retaining the in-phase reflection behavior of EBG structures. They are basically made up of an FSS layer over a ground plane. Using the characteristics of the frequency-dependent reflection phases, AMC structures can generate $\mathrm{CP}$ waves from linear polarized (LP) waves [7]. The designs in [6] and [7] exhibited dual and single narrow axial ratio (AR) bandwidths $(\mathrm{AR} \leq 3 \mathrm{~dB})$ respectively, while the design in [5] offered an AR bandwidth of $20.4 \%$. Since the frequency band covering all SNS is at least $1.16-1.60 \mathrm{GHz}$ [1], i.e. $32 \%$ fractional bandwidth, none of the mentioned designs are adequate. Owing to the tunable capacitance of their active components [8-13], AMC can improve the bandwidth of the reflection phase of CP antennas [14].

This paper presents a novel active FSS and its application to frequency tunable CP antennas. The active FSS layer is an improved version to the one presented by the authors in [15]. An active AMC structure based on this design is presented. The active AMC exhibits dual-polarized tunable reflection phases versus frequency. As with the active FSS, it can tune two orthogonal polarizations incidentally. A coplanar waveguide (CPW) fed monopole is placed on the AMC, and its frequency and polarization characteristics are dynamically modified. Simulation studies were implemented using CST Microwave Studio. Tuning the capacitances of varactor diodes, $\mathrm{CP}$ waves can be realized between the $1.15 \mathrm{GHz}-1.60 \mathrm{GHz}$ necessary to cover all SNS. Owing to the symmetry of the design, agile switching between right hand $\mathrm{CP}$ (RHCP) and left hand $\mathrm{CP}$ (LHCP) polarizations is also obtained.

\section{SIMPLIFIED DUAL POLARIZED ACTIVE FSS}

A tunable patch-form FSS capable of modifying the frequency response in two polarisations was presented in [15]. A new simplified design is shown in Fig.1. It consists of a double layer structure with identical patch elements at the top and bottom layer and with active components arranged to achieve independent control in two polarisations. Fig. 1 a) and b) illustrate the top and the bottom layers of the FSS with the corresponding diodes and resistors. The two metallic layers sandwiching a $0.05 \mathrm{~mm}$ thick dielectric substrate. 


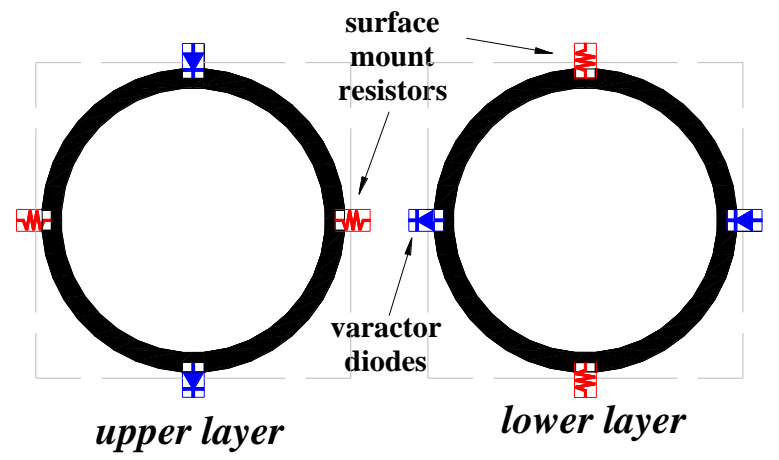

(a)

(b)

Fig.1. Unit cell of the dual polarised active FSS

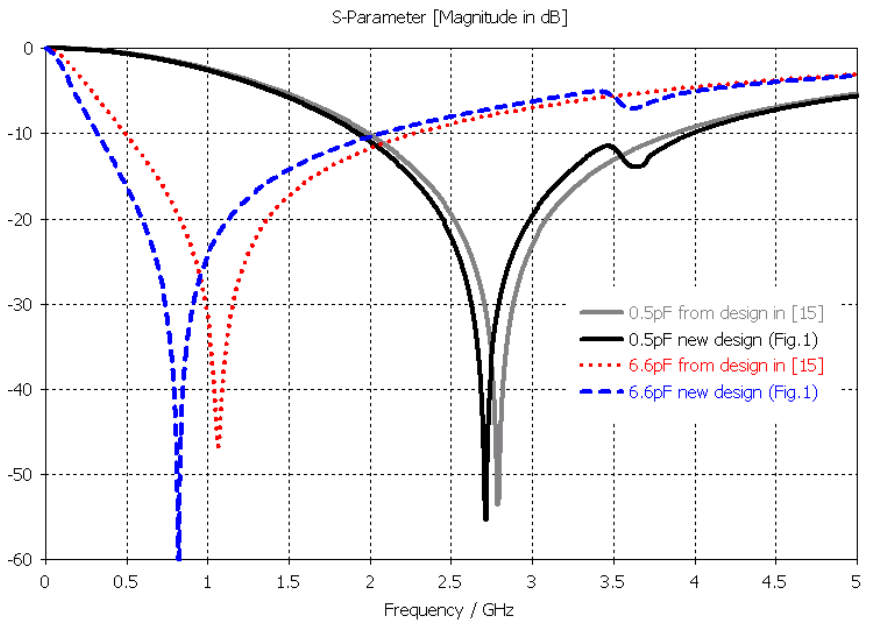

Fig. 2 Transmission responses, dB

The design operates in a similar way to that in [15] but uses half the number of resistors per cell (from 4 to 2), thus decreasing resistive losses and increasing efficiency.

The other main difference between designs in [15] and the design in Fig. 1 is the way they are controlled. In Fig.1, the change in the capacitance of the varactors in the y-axis (upper layer) is carried out through a change in the voltage applied trough the resistors in the $\mathrm{x}$-axis (upper layer). In [15], the change in capacitance of the diodes in the $y$-axis is through voltage in the resistors in the same y-axis. In both cases, the voltage drop caused by the very small current flowing across the diodes can be neglected, especially when the array size is not large [15-17]. Fig. 2 shows the simulated transmission response for the two designs. Both designs present wideband responses. When the capacitance of the diodes is $0.5 \mathrm{pF}$, the new design resonates at $2.7 \mathrm{GHz}$. This is about $3.5 \%$ lower frequency than that for [15]. When the capacitance is $6.6 \mathrm{pF}$, the new design resonates at about $0.8 \mathrm{GHz}, 20 \%$ lower than in [15]. Therefore not only the new design reduces the number of resistor employed but also decreases the resonant frequency and increases the frequency range of operation.

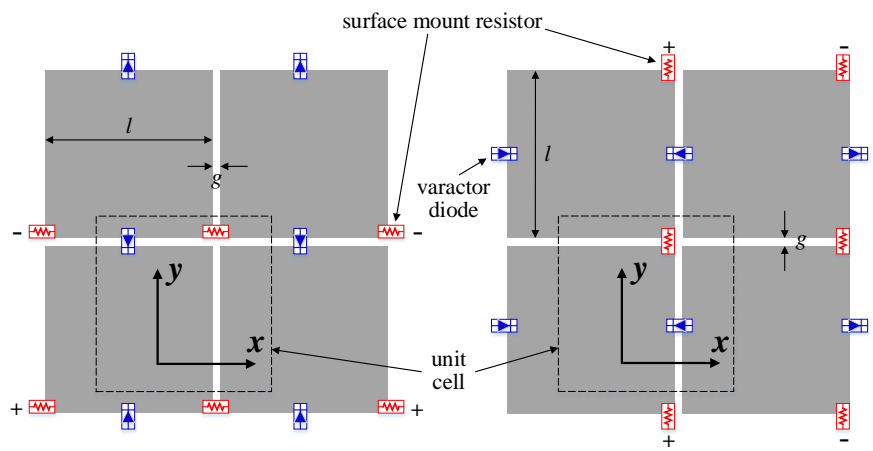

(a)

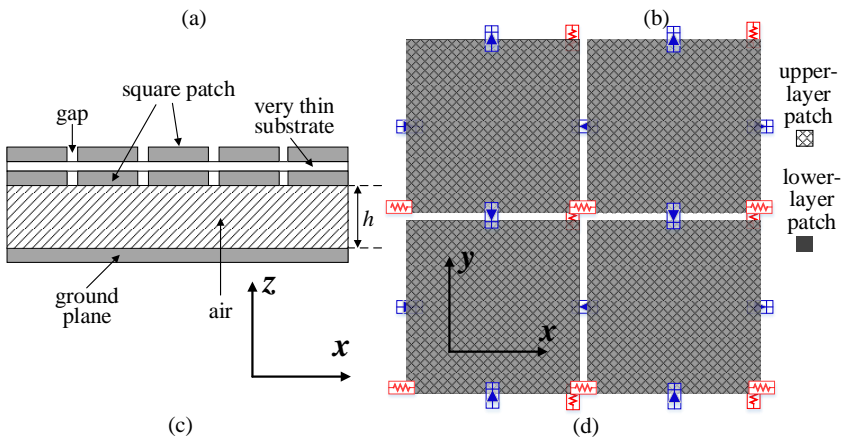

Fig. 3. Unit cells of the dual polarized active AG structure with varactors and resistors. (a) Upper layer (b) Lower layer (c) Magnified cross-sectional view (d) Alignment of the upper and lower layers.

\section{DUAL POLARIZED ACTIVE AMC STRUCTURE}

The same biasing technique can be applied to an AMC made of square patches, as illustrated in Fig.3 [18]. The periodic FSS layer and the ground plane are spaced by an air layer, with a height of $h$. A polyester substrate $\left(\varepsilon_{\mathrm{r}}=3\right)$ of $0.05 \mathrm{~mm}$ thickness with metal cladding on its two sides is used to form the FSS, and the square patches are printed on both sides. As seen in Fig. 3 (d), the upper and the lower layer are overlapped in X-y plane.

The varactor diodes were BB857, modeled as a series RLC circuit in simulation. According to the datasheet, the series resistance and inductance are $1.5 \Omega$ and $0.6 \mathrm{nH}$ respectively, and the variable capacitance can be tuned from $0.55 \mathrm{pF}$ to $6.6 \mathrm{pF}$. In the following, the uniform capacitances of varactors on the upper and lower layers are defined as $C_{1}$ and $C_{2}$ respectively.

In order to demonstrate the polarization-independent and symmetrical tuning ability, $C_{1}$ and $C_{2}$ were varied from $0.55 \mathrm{pF}$ to $3.55 \mathrm{pF}$ in simulation. Other parameters were as follows: $l=21 \mathrm{~mm}, g=1 \mathrm{~mm}, h=5 \mathrm{~mm}$. As shown in Fig. 4(a), when $C_{1}$ is fixed to $0.55 \mathrm{pF}$ while $C_{2}$ is decreased, the phase curve of the $\mathrm{x}$ polarization shift towards higher frequencies, while the phases of the y-polarization remains stable. Hence, the independent tuning capability for dual polarizations is verified. It is also indicated that the reflection phases for the $\mathrm{x}$ - and y-polarized waves agree very well with each other when both capacitances are $0.55 \mathrm{pF}$, demonstrating the tuning symmetry for the dual polarizations. Similarly, the phase stability of tuning for the orthogonal polarization in lower frequencies is illustrated in Fig. 4(b), as well as the polarization symmetry. 


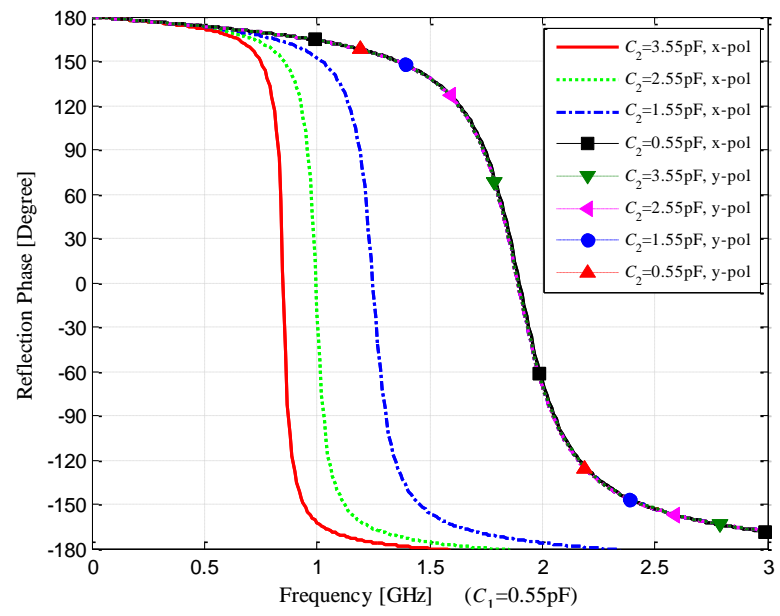

(a)

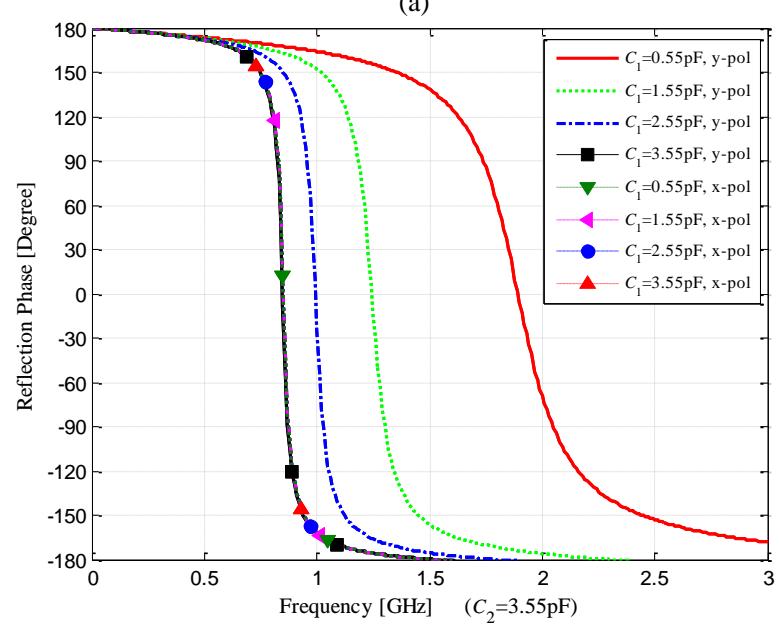

(b)

Fig. 4. Tunable reflection phases for dual polarizations (a) $C_{1}$ is fixed, $C_{2}$ is varied (b) $C_{2}$ is fixed, $C_{1}$ is varied

\section{WIDEBAND RECONFIGURABLE CP ANTENNA USING ACTIVE AMC STRUCTURE}

\section{A. Configuration}

The schematic diagram of the antenna is shown in Fig. 5, in which the resistors are hidden for clarity. The AMC consists of $6 \times 6$ unit cells, and a CPW monopole antenna rotated by $45^{\circ}$ with respect to the EBG lattice is placed above the upper patch layer, with a distance of $h^{\prime}$. The dimensions and reflection coefficient of the monopole in free space are presented in Fig. 6. The substrate of the monopole is Rogers 5870, with $\varepsilon_{r}$ of 2.33 and the thickness of $0.787 \mathrm{~mm}$.

Similar to the dipole used in [7], the CPW monopole radiates omnidirectional LP waves in the plane of $\varphi=135^{\circ}$ in Fig. 3, but offering a broadband input matching. According to the composition principle of the directly radiated wave and the reflected wave described in [7], the LP waves can be converted to $\mathrm{CP}$ waves over a wide band. The theoretical condition to generate $\mathrm{CP}$ waves is the reflection phase for one-polarized wave equals to $90^{\circ}$, while the phase for the other polarization is $-90^{\circ}$.

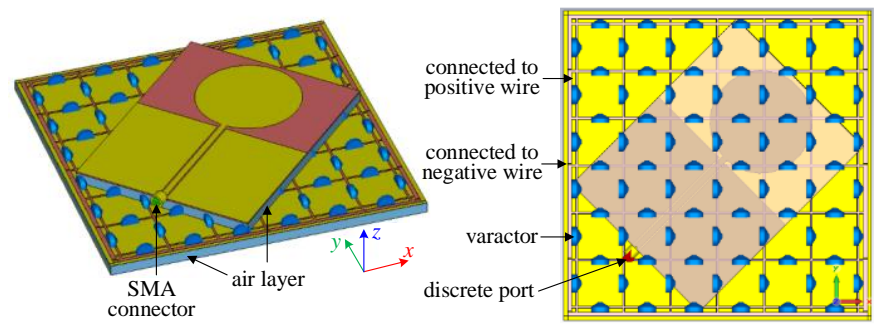

(a)

(b)

Fig. 5. Schematic diagram of the antenna (a) Perspective view (b) Top view

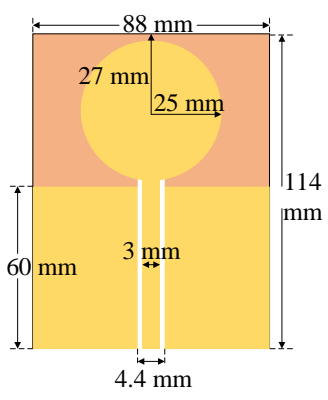

(a)

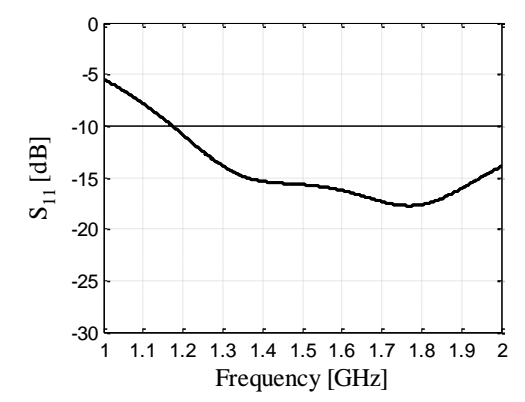

(b)
Fig. 6. The CPW monopole antenna (a) Top view (b) Reflection coefficient

In order to obtain accurate prediction of the antenna performances, the entire structure including the varactor diodes should be modeled in CST, rather than simply referring to the values of $C_{1}$ and $C_{2}$ obtained in the unit cell simulation.

\section{B. Frequency Tunable Capability}

According to the datasheet, the lowest tunable capacitance is $0.55 \mathrm{pF}$, corresponding to the highest frequency acquiring $\mathrm{AR}$. Hence by fixing $C_{1}=0.55 \mathrm{pF}, h=h{ }^{\prime}=5 \mathrm{~mm}$, and optimizing $C_{2}$ and the side length $l$ of the square patch of the AG structure, a fairly low broadside AR was obtained at the target frequency, $1.60 \mathrm{GHz}$. The optimal values were: $C_{2}=1.25 \mathrm{pF}$, and $l=22 \mathrm{~mm}$.

As aforementioned, the reflection phase curves for dual polarizations can be tuned continuously towards lower frequencies, as the capacitances increase. To demonstrate this tuning capability, the AR curves are tuned to reach a low value at four sampling frequencies from $1.15 \mathrm{GHz}$ to $1.60 \mathrm{GHz}$ respectively. Fig. 7 presents the simulated ARs and reflection coefficients. It can be seen that not only satisfactory ARs are reached at the four frequencies, but also $S_{11}$ less than $-10 \mathrm{~dB}$ are obtained respectively, owing to the wideband matching characteristics of the radiating monopole antenna. The radiation patterns at each frequency are presented in Fig. 8, in which low cross-polarizations within a wide range of angles can be observed. In order to clearly show the varying tendency of $C_{1}$ and $C_{2}$ in the tuning process, their values to realize $\mathrm{CP}$ characteristics at the four frequencies are plotted in Fig. 8. It is found that both $C_{1}$ and $C_{2}$ are in approximately linear variation over the band between 1.15$1.60 \mathrm{GHz}$, which can be a helpful guide to efficiently tune the $\mathrm{AR}$ frequency onto any other frequencies over the frequency range. The directivity and gain at each frequency also presented in Fig. 9, and the gains are found to drop dramatically in lower frequencies. This is due to the low quality factor of the varactor diodes when series capacitance is high. 


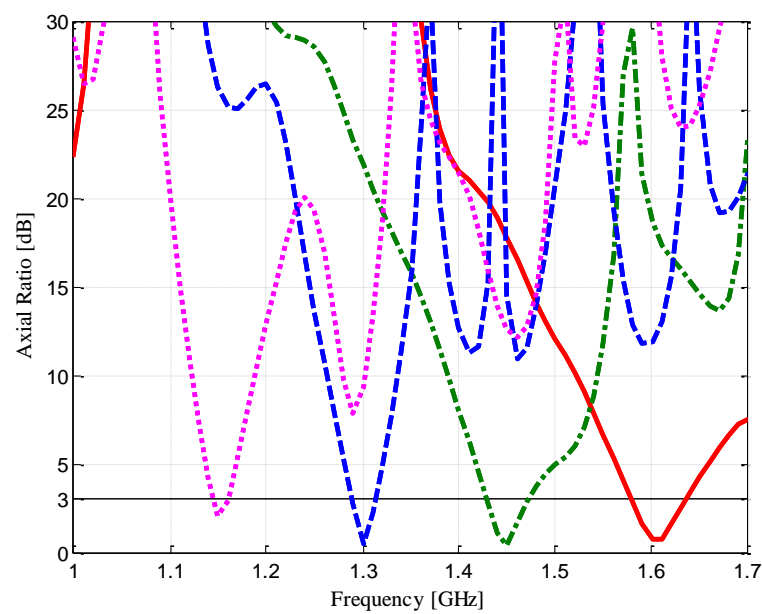

(a)

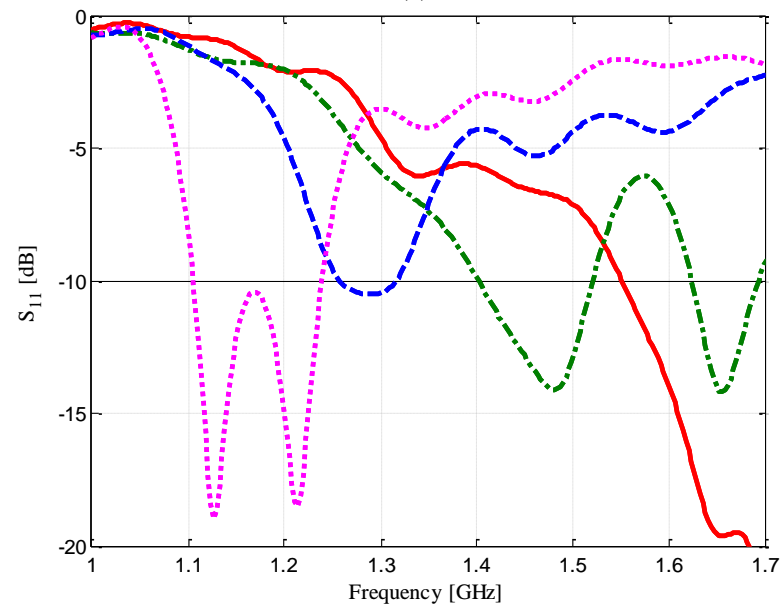

(b)

$C_{1}[\mathrm{pF}] \quad 0.55 \quad 1.18 \quad 1.95 \quad 2.48$

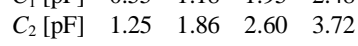

Fig. 7. Illustration of tuning capability (a) AR (b) reflection coefficients
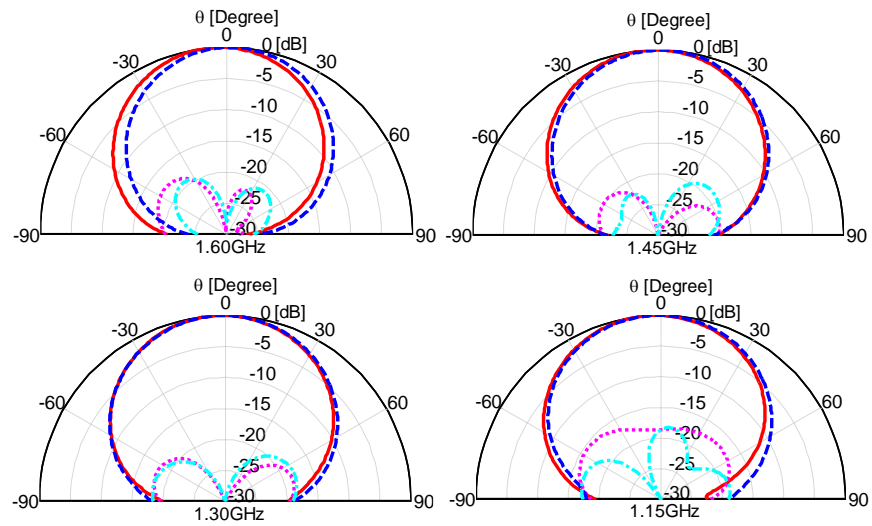

— LHCP in $x-z$ plane

....... RHCP in $x$-z plane

- - - LHCP in $y-z$ plane

$-\cdot-\cdot-$ RHCP in $y-z$ plane

Fig. 8. Radiation patterns at four demonstrating frequencies

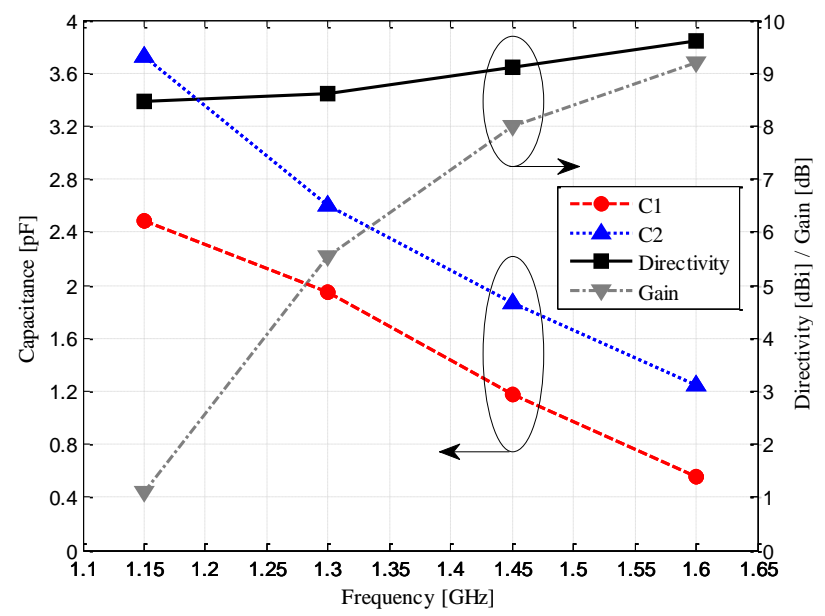

Fig. 9. Capacitances, directivity and gain at each frequency

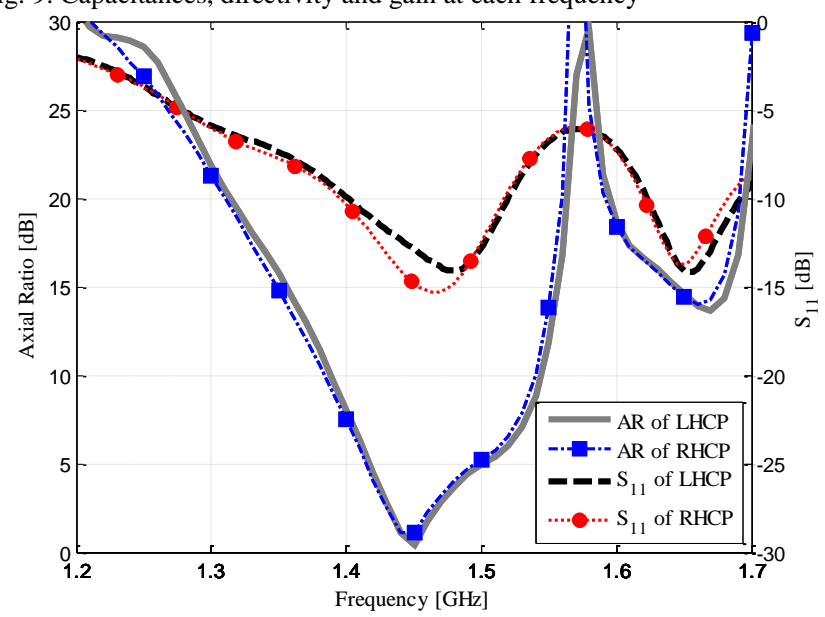

Fig. 10. Switchable capability in terms of AR and $S_{11}$ versus frequency

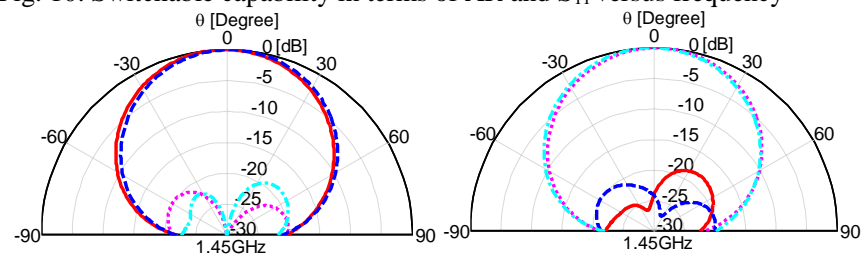

LHCP in $x$-z plane
$\ldots \ldots$ RHCP in $x$-z plane
---- LHCP in $y-z$ plane
.--- RHCP in $y$-z plane

Fig. 11. Switchable capability in terms of radiation patterns

\section{Polarization Switchable Capability}

In the previous sub-section, $C_{1}$ remained less than $C_{2}$, so LHCP was the co-polarization at all cases. Considering the symmetry of the reflection phases for dual polarizations and the antenna configuration, LHCP can be changed into RHCP by simply swapping $C_{1}$ and $C_{2}$. To conserve space, the case of $1.45 \mathrm{GHz}$ is taken as an example. According to Fig. 5(a), LHCP was realized at $1.45 \mathrm{GHz}$ when $C_{1}=1.18 \mathrm{pF}$ and $C_{2}=1.86 \mathrm{pF}$. Fig. 10 presents the AR and $\mathrm{S}_{11}$ curves before and after swapping $C_{1}$ and $C_{2}$, showing good agreements between the LHCP and RHCP cases. The radiation patterns at $1.45 \mathrm{GHz}$ of the two cases are shown in Fig. 11. In the left figure the co-polarization is 
LHCP, while in the right figure it is RHCP. Hence, the capability of the switchable $\mathrm{CP}$ polarization is clearly demonstrated.

\section{CONCLUSION AND DISCUSSION}

A new simplified design for an active frequency selective surfaces has been presented. The design is dual polarized and can modify the transmission responses of two polarization independently. The design has the same reconfigurable capability to the one described in [15] but uses half the number of resistors, potentially decreasing resistive losses and increasing efficiency. In addition, it resonates at lower frequency and cover a wider range of frequencies.

This biasing technique has been successfully applied to the FSS layer of artificial magnetic conductors (AMC) [18]. As with the active FSS, the AMC structure exhibits tunable capability of the reflection phases for dual polarizations, and tunings for the $\mathrm{x}$ - and the $\mathrm{y}$ - polarizations are symmetrical and independent. By tuning the varactor capacitances in the two directions together, high-quality axial ratios (ARs) can be obtained over a wide band covering the frequencies of all satellite navigation systems. As a demonstration, $\mathrm{CP}$ characteristics at four equally spaced frequencies from $1.15 \mathrm{GHz}$ to $1.60 \mathrm{GHz}$ have been presented, and input reflection coefficients of less than $-10 \mathrm{~dB}$ are have been obtained at each frequency. By swapping the capacitances of the varactors on the two patch layers, the left hand circular polarization (LHCP) can be converted to right hand circular polarization (RHCP) straightforwardly. The antenna efficiency is found to be significantly reduced in lower frequencies, due to the series resistance and the high capacitance of the varactor diodes.

\section{REFERENCES}

[1] S. Gao, Q. Luo, and F. Zhu, Circularly polarized antennas: John Wiley \& Sons, 2013.

[2] G. Massie, M. Caillet, M. Clénet, and Y. M. Antar, "A new wideband circularly polarized hybrid dielectric resonator antenna," Antennas and Wireless Propagation Letters, IEEE, vol. 9, pp. 347-350, 2010.

[3] L. Wang, Y.-X. Guo, and W. Sheng, "Tri-band circularly polarized annular slot antenna for GPS and CNSS applications," Journal of Electromagnetic Waves and Applications, vol. 26, pp. 1820-1827, 2012.
[4] S.-H. Chang and W.-J. Liao, "A novel dual band circularly polarized GNSS antenna for handheld devices," Antennas and Propagation, IEEE Transactions on, vol. 61, pp. 555-562, 2013.

[5] T. Nakamura and T. Fukusako, "Broadband design of circularly polarized microstrip patch antenna using artificial ground structure with rectangular unit cells," Antennas and Propagation, IEEE Transactions on, vol. 59, pp. 2103-2110, 2011.

[6] H. Yi and S.-W. Qu, "A Novel Dual-Band Circularly Polarized Antenna Based on Electromagnetic Band-Gap Structure," 2013.

[7] F. Yang and Y. Rahmat-Samii, "A low profile single dipole antenna radiating circularly polarized waves," Antennas and Propagation, IEEE Transactions on, vol. 53, pp. 3083-3086, 2005.

[8] L. Boccia, F. Venneri, G. Amendola, and G. Di Massa, "Application of varactor diodes for reflectarray phase control," in Antennas and Propagation Society International Symposium, 2002. IEEE, 2002, p. 132.

[9] D. Sievenpiper and J. Schaffner, "Beam steering microwave reflector based on electrically tunable impedance surface," Electronics Letters, vol. 38, pp. 1237-1238, 2002.

[10] D. F. Sievenpiper, J. H. Schaffner, H. J. Song, R. Y. Loo, and G. Tangonan, "Two-dimensional beam steering using an electrically tunable impedance surface," Antennas and Propagation, IEEE Transactions on, vol. 51, pp. 2713-2722, 2003.

[11] C. Mias and J. H. Yap, "A varactor-tunable high impedance surface with a resistive-lumped-element biasing grid," Antennas and Propagation, IEEE Transactions on, vol. 55, pp. 1955-1962, 2007.

[12] H.-J. Lee, R. Langley, and K. Ford, "Tunable active EBG," in Antennas and Propagation, 2007. EuCAP 2007. The Second European Conference on, 2007, pp. 1-4.

[13] H. Lee, K. Ford, and R. Langley, "Dual band tunable EBG," Electronics Letters, vol. 44, pp. 392-394, 2008.

[14] B. Liang, B. Sanz-Izquierdo, E. A. Parker, and J. C. Batchelor, "A Frequency and Polarization Reconfigurable Circular Polarized Antenna Using Active EBG Structure for Satellite Navigation”, IEEE Trans. Antennas Propag, Vol. 63, No.1, pp. 33 - 40, 2015.

[15] B. Sanz-Izquierdo and E. Parker, "Dual Polarized Reconfigurable Frequency Selective Surfaces," Antennas and Propagation, IEEE Transactions on, vol. 62, pp. 764 - 771, 2014.

[16] B. Sanz-Izquierdo, E. Parker, J.-B. Robertson, and J. Batchelor, "Tuning patch-form FSS," Electronics Letters, vol. 46, pp. 329-330, 2010.

[17] C. Mias, "Varactor-tunable frequency selective surface with resistivelumped-element biasing grids," Microwave and Wireless Components Letters, IEEE, vol. 15, pp. 570-572, 2005.

[18] B. Liang, B. Sanz-Izquierdo, E. A. Parker, and J. C. Batchelor, "Frequency tunable and circular polarization switchable antenna using dual polarized active artificial ground structure", 9th European Conference on Antennas and Propagation, Lisbon, pp. 1 - 5, 2015. 